\title{
Pathogenicity of an H5N1 Highly Pathogenic Avian Influenza Virus Isolated in the 2010-2011 Winter in Japan to Mandarin Ducks
}

\author{
Kosuke SODA ${ }^{1)}$, Tatsufumi USUI ${ }^{1,2)}$, Yukiko $\mathrm{UNO}^{2)}$, Kumiko YONEDA ${ }^{3)}$, Tsuyoshi YAMAGUCHI ${ }^{1,2)}$ and \\ Toshihiro $\mathrm{ITO}^{1,4) *}$ \\ 1) Avian Zoonosis Research Center, Faculty of Agriculture, Tottori University, 4-101 Koyama-Minami, Tottori 680-8553, Japan \\ 2) Laboratory of Veterinary Hygiene, Department of Veterinary Medicine, Faculty of Agriculture, Tottori University, 4-101 Koyama- \\ Minami, Tottori 680-8553, Japan \\ 3) Japan Wildlife Research Center, 3-10-10 Taitou-ku, Tokyo 110-8676, Japan \\ 4) Laboratory of Veterinary Public Health, Department of Veterinary Medicine, Faculty of Agriculture, Tottori University, 4-101 Koyama- \\ Minami, Tottori 680-8553, Japan
}

(Received 5 November 2012/Accepted 22 December 2012/Published online in J-STAGE 15 January 2013)

ABSTRACT. Widespread outbreaks of highly pathogenic avian influenza (HPAI) caused by H5N1 viruses occurred in wild birds in Japan from 2010-2011. Forty out of 63 deceased wild birds belonged to the order Anseriformes, and mandarin duck was one of the dominant species. To estimate the risk of mandarin ducks as a source of virus infection in the environment, we examined the pathogenicity of a causal H5N1 HPAI virus to mandarin ducks. About half of the mandarin ducks died by inoculation with $10^{7.0} \mathrm{TCID} \mathrm{D}_{50}$ of $\mathrm{A} / \mathrm{mandarin}$ duck/ Miyazaki/22M807-1/2011 (H5N1). Viruses were mainly recovered from the trachea of the ducks sacrificed at three days post inoculation (d.p.i.). Viruses were recovered from the laryngopharyngeal swabs of the observation group until 5 d.p.i. In ducks that died at the late phase of infection, viruses were detected in the systemic organs, such as lung, kidney and colon. Together, these results showed that the H5N1 HPAI viruses, which belonged to clade 2.3.2.1 and are mainly circulating in East Asia, were lethal to mandarin ducks, indicating that mandarin ducks have the potential to disseminate the virus to other bird species. Therefore, wild birds should be kept out of poultry farms to prevent HPAI outbreaks in the future.

KEY WORDS: H5N1, influenza virus, mandarin duck, pathogenicity.

doi: 10.1292/jvms.12-0487; J. Vet. Med. Sci. 75(5): 619-624, 2013

Until now, H5N1 highly pathogenic avian influenza viruses (HPAIVs) have spread to domestic poultry and wildlife in 63 countries in Asian, Europe, Middle East and Africa since late 1996 when the virus, recognized as the precursor of present circulating viruses, was isolated from a goose in Guangdong province, China [20, 21]. These incidents have increased the possibility of further spread of HPAIVs to humans and rare species of animals.

In Japan, the outbreaks caused by H5N1 HPAIVs occurred in chicken farms in 2003-2004 [9] and 2007. These appear to be the first HPAI outbreaks since 1925 [15]. In addition, H5N1 HPAIVs were isolated from wild birds, such as jungle crow (Corvus macrorhynchos), mountain hawk-eagle (Nisaetus nipalense) and whooper swan (Cygnus cygnus) in 2004, 2007 and 2008, respectively [13, 16, 17]. These outbreaks were controlled by depopulation of chickens in the relevant farms, intensive surveillance and improved biosecurity measures. Since then, continuous surveillance has confirmed that poultry and wild birds in Japan have been free of HPAIV infection.

\footnotetext{
*Correspondence to: Ito, T., Laboratory of Veterinary Public Health, Department of Veterinary Medicine, Faculty of Agriculture, Tottori University, 4-101 Koyama-Minami, Tottori 680-8553, Japan.

e-mail: toshiito@muses.tottori-u.ac.jp

(C)2013 The Japanese Society of Veterinary Science
}

However, many outbreaks occurred in Japan during the 2010-11 winter; H5N1 HPAIVs were isolated from 63 wild birds including migrating and resident birds, and caused HPAI outbreaks in 24 chicken farms by the end of March $[12,18]$. Around the same time, the HPAI outbreaks occurred in both domestic and wild bird populations in South Korea [7]. Just before these outbreaks, H5N1 HPAIVs were isolated from fecal samples of migrating ducks in the northernmost part of Japan in autumn of 2010 [4], and also from the healthy mallard ducks captured in South Korea in 2010 [6]. These reports support the assumption that migratory waterfowl flying from their nesting lakes in Siberia, a natural reservoir of influenza A viruses, could play a role in dissemination of H5N1 HPAIVs to domestic poultry and resident bird species.

In the 2010-11 outbreaks in Japan, 17 wild bird species were confirmed to be infected with H5N1 HPAIV $[10,12]$. Forty out of 63 deceased wild birds belonged to the order Anseriformes. Of these 40 birds, 12 were tufted ducks (Aythya fuligula) and 11 were mandarin ducks (Aix galericulata). Mandarin duck was also dominant among the wild birds with HPAIV in the outbreaks in Korea [7].

Mandarin ducks are indigenous to East Asian countries, and are recognized as sedentary birds in Japan [3]. Unlike other Anseriformes, they breed in densely wooded areas, near shallow lakes, marshes or ponds. The diet of mandarin ducks changes seasonally. In autumn and winter, they mainly eat acorns and grains. In the breeding season, they eat mostly 


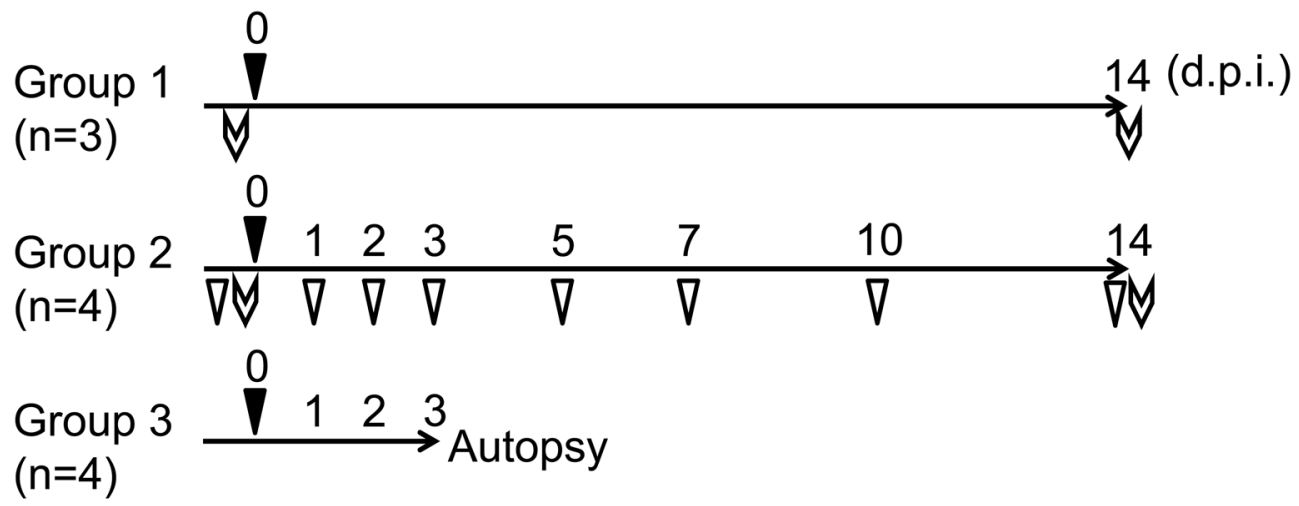

\section{Virus Inoculation $\quad \nabla$ Swab sampling $\quad$ Blood sampling}

Fig.1. Experimental design of H5N1 HPAIV inoculation to mandarin ducks. All mandarin ducks were intranasally inoculated with $10^{7.0} \mathrm{TCID}_{50}$ of A/mandarin duck/Miyazaki/22M807-1/2011 (H5N1). Three birds (Group 1) were observed for clinical signs for 14 days. Laryngopharyngeal and cloacal swabs of 4 other birds (Group 2) were sampled at 1, 2, 3, 5, 7, 10 and 14 days post inoculation. In Groups 1 and 2, specific antibodies against homologous virus after 14 days of infection were detected in serum by HI test. The other 4 ducks (Group 3 ) were euthanized at three days post inoculation, and their tissues were collected.

insects, snails, fish and aquatic plants. This seasonal change of life style increased the opportunity to share territory with migrating and raptorial birds and to get close to poultry farms. In fact, flocks of mandarin ducks were frequently observed around chicken farms located in mountain area [10]. In one case, a mandarin duck that died of H5N1 HPAIV infection was found near a chicken farm in which an HPAI outbreak occurred only two days before.

To clarify the transmission route of H5N1 HPAIV among domestic and wild bird species, the pathogenicity of viruses for mandarin ducks needs to be examined. Kwon et al. reported the pathobiology of A/chicken/Korea/IS/2006 (H5N1), whose hemagglutinin (HA) gene was classified into clade 2.2, in mandarin ducks [8]. However, pathogenicity of the current circulating H5N1 HPAIVs belonged to Clade 2.3.2.1 worldwide is still unknown. In the present study, mandarin ducks were inoculated with $\mathrm{A} /$ mandarin duck/ Miyazaki/22M807-1/2011 (H5N1) [12] isolated during the $2010-11$ outbreaks in Japan, and its pathogenicity was evaluated.

\section{MATERIALS AND METHODS}

Virus: A/mandarin duck/Miyazaki/22M807-1/2011 (H5N1) (M807) was isolated from the mixture of tracheal and cloacal swabs of the mandarin duck died in Miyazaki city located in Kyusyu region of Japan [12]. Accession numbers of the gene sequences of M807 are AB677872AB677879. Viruses were propagated in ten-day-old embryonated chicken eggs for $48 \mathrm{hr}$ at $35^{\circ} \mathrm{C}$.

Animals: Eleven 3- to 7-year-old mandarin ducks (4 male birds and 7 female birds) were kindly provided by Inokashira Park Zoo (Tokyo, Japan) in agreement with the Japanese
Ministry of the Environment. The animals were shipped by air to Tottori University, confirmed influenza virus-negative by antigen detection kit, ESPLINE INFLUENZA A \& B-N (Fujirebio Inc., Tokyo, Japan) and then used for experiments. Experimental infection: The 11 ducks were intranasally inoculated with $200 \mu \mathrm{l}$ of allantoic fluid containing M807 at $10^{7.0} 50 \%$ tissue culture infectious dose $\left(\mathrm{TCID}_{50}\right)$, and divided into three groups (Fig. 1). The ducks in Group $1(n=3$; \#01-\#03) and Group $2(\mathrm{n}=4$; \#04-\#07) were observed for signs of disease at 24-hr intervals for 14 days. The Group 2 ducks were also checked for viral shedding in laryngopharyngeal and cloacal swabs at several times. The swabs were collected in $2 \mathrm{ml}$ of nutrient broth medium (Nissui Pharmaceutical, Tokyo, Japan) with $10 \mathrm{mg}$ streptomycin (Meiji Seika Pharma, Tokyo, Japan) and 10,000 units of penicillin G (Meiji Seika Pharma). At the end of the 14-day period, the ducks in Groups 1 and 2 were also checked for specific antibodies in the serum against homologous virus by hemagglutinin inhibition (HI) test [5]. The ducks in Group 3 $(\mathrm{n}=4 ; \# 08$-\#11) were used for tissue samples at 3 days post inoculation (d.p.i.). After blood collection, the birds were euthanized and dissected. Brain, trachea, lung, kidney and colon were aseptically collected and homogenized to make $10 \%(\mathrm{w} / \mathrm{v})$ suspensions in nutrient broth medium with antibiotics. Infectious viral titers in the collected samples were determined in Madin-Derby Canine Kidney (MDCK) cells by the method of Reed and Muench [11].

All animal experiments were carried out in self-contained isolator units (CLEA Japan, Tokyo, Japan) at a biosafety level 3 facility at Avian Zoonosis Research Center, Tottori University, Japan. The experiments were performed according to the guidelines of the institutional animal care and use committee of Tottori University (approved number: 11-T-98). 
Table 1. Clinical signs and antibody responses of the mandarin ducks intranasally inoculated with $10^{7.0} \mathrm{TCID}_{50}$ of $\mathrm{A} / \mathrm{mandarin}$ duck/Miyazaki/22M807-1/2011 (H5N1)

\begin{tabular}{|c|c|c|c|c|c|c|c|c|c|c|c|c|c|c|}
\hline & \multirow{2}{*}{ ID } & \multicolumn{11}{|c|}{ Clinical signs } & \multicolumn{2}{|c|}{ Serum HI titer } \\
\hline & & $0-2 d$ & $3 \mathrm{~d}$ & $4 d$ & $5 \mathrm{~d}$ & $6 \mathrm{~d}$ & $7 d$ & $8 \mathrm{~d}$ & $9 \mathrm{~d}$ & $10 \mathrm{~d}$ & $11 \mathrm{~d}$ & $12-14 d$ & Pre & Post \\
\hline \multirow{3}{*}{ Group 1} & $\# 01$ & - & - & - & - & - & - & - & - & - & dead & & $<2$ & $\mathrm{NA}^{\mathrm{a})}$ \\
\hline & $\# 02$ & - & - & - & - & - & - & - & - & - & - & - & $<2$ & 16 \\
\hline & $\# 03$ & - & - & - & - & - & - & - & - & - & - & - & $<2$ & $<2$ \\
\hline \multirow{4}{*}{ Group 2} & $\# 04$ & - & - & - & - & - & dead & & & & & & $<2$ & NA \\
\hline & $\# 05$ & - & - & - & - & - & - & - & - & - & + & - & 2 & 16 \\
\hline & $\# 06$ & - & - & - & + & - & ++ & dead & & & & & $<2$ & NA \\
\hline & $\# 07$ & - & - & - & - & - & - & - & - & - & + & - & $<2$ & 16 \\
\hline \multirow{4}{*}{ Group 3} & $\# 08$ & - & autopsy & & & & & & & & & & $<2$ & NA \\
\hline & $\# 09$ & - & autopsy & & & & & & & & & & $<2$ & NA \\
\hline & $\# 10$ & - & autopsy & & & & & & & & & & $<2$ & NA \\
\hline & $\# 11$ & - & autopsy & & & & & & & & & & $<2$ & NA \\
\hline
\end{tabular}

a) Not applicable.

\section{RESULTS}

Serological tests of the provided mandarin ducks: All but one of the ducks $(\# 05,2 \mathrm{HI})$ were free of antibodies to H5 HA antigen (Table 1). To examine the pathogenicity of the H5N1 HPAI virus for mandarin ducks having low titer of antibody against H5 HA, \#05 was also used for the subsequent studies.

Intranasal pathogenicity of M807 strain in mandarin ducks: One out of three ducks in Group 1 died at 11 d.p.i., although clinical signs were not observed until day 10 (Table 1). The other 2 ducks survived for 14 days without showing any clinical signs, and one of them seroconverted. In Group 2, one bird died at 7 d.p.i., and another died at 8 d.p.i. Notably, \#06 presented with apparent symptoms, such as decreased appetite, gait disorder, dysstasia and depression at 7 d.p.i. The others showed temporary and mild clinical signs at 11 d.p.i. and survived for 14 days. Antibodies against the homologous virus were detected in the sera of both surviving ducks at 14 d.p.i. The birds in Group 3 did not show any signs of disease during the experimental period.

Virus shedding: The virus titers in the laryngopharyngeal and cloacal swabs of the birds in Group 2 were determined at the days shown in Fig. 1. Viruses were recovered from the laryngopharyngeal swabs of all birds at 1 d.p.i., and the duration of viral shedding was 2 to 5 days (Fig. 2A). Virus was recovered from the cloacal swabs from only two birds (\#04 and \#07), and their titers were relatively low (Fig. 2B). For bird \#06, which died at 8 d.p.i., the virus titer in the laryngopharyngeal swab was high at 5 d.p.i. when the symptoms began (Table 1). For \#04, viruses were recovered from the cloacal swabs (but not the laryngopharyngeal swabs) at 5 d.p.i. and at 7 d.p.i., the day it died.

Viral replication in mandarin ducks: In Group 3, viruses were recovered mainly from the trachea, and also from the other tissues, except for the brain and the lung (Fig. 3). By contrast, viruses were detected in the lung samples of two birds, \#06 and \#01, which died at the late phase of infection, while no virus was isolated from the trachea of the dead birds in Groups 1 and 2.

\section{DISCUSSION}

In the present study, it was evaluated that pathogenicity of H5N1 HPAIV for mandarin ducks, suspected to be potential source of infection. The death rate in Groups 1 and 2 was $50 \%$ and $33 \%$, respectively (Table 1 ). In other words, A/ mandarin duck/Miyazaki/22M807-1/2011 (H5N1) showed moderate pathogenicity to mandarin ducks as compared with other bird species; the virus, isolated in 2010-11 outbreaks in Japan, was not lethal to domestic ducks, while it showed $100 \%$ mortality to chickens $[4,18]$. Three of the ducks that survived the 14-day experimental period including $\# 05$, in which antibody against $\mathrm{H} 5 \mathrm{HA}$ of the challenged strain was detected, showed increased HI antibody titers, suggesting that mandarin ducks which experienced influenza virus infection in the past could be reinfected with H5N1 HPAIVs. The pre- and post- serum HI titers of \#05 against non-pathogenic H5 virus, A/tundra swan/Shimane/3211A001/2011 $(\mathrm{H} 5 \mathrm{~N} 2)$, were $16 \mathrm{HI}$ and $8 \mathrm{HI}$, respectively (data not shown). It was formerly reported that there were some differences in antigenicity of H5 HAs between HPAIV and non-pathogenic viruses $[12,14,22]$. Therefore, $\# 05$ was thought to be previously exposed to non-pathogenic $\mathrm{H} 5$ virus.

In Group 2, the virus was mainly recovered from the laryngopharyngeal swabs rather than by the cloacal swabs (Fig. 2). A similar result was reported in mandarin ducks infected with a virus in clade 2.2 [8]. The virus titers in laryngopharyngeal swab samples were up to $10^{3.5} \mathrm{TCID}_{50} / \mathrm{m} l$, suggesting that the transmission of the virus among mandarin duck flocks is unlikely to occur. Actually, no mass death of mandarin ducks was observed in the 2010-11 outbreaks in Japan [12]. On the other hand, it was previously reported that chicken lethal doses of the HPAIVs isolated in Japan in 2010-2011 were $10^{3}-10^{4.3} 50 \%$ egg infectious doses / $\mathrm{m} l$ [18], which were comparable to the amount of the virus detected in the laryngopharyngeal swabs of the mandarin ducks in the present study. Thus, mandarin ducks were suspected to be a potential source of infection to chickens.

In Group 3, viruses were mostly recovered from the tra- 

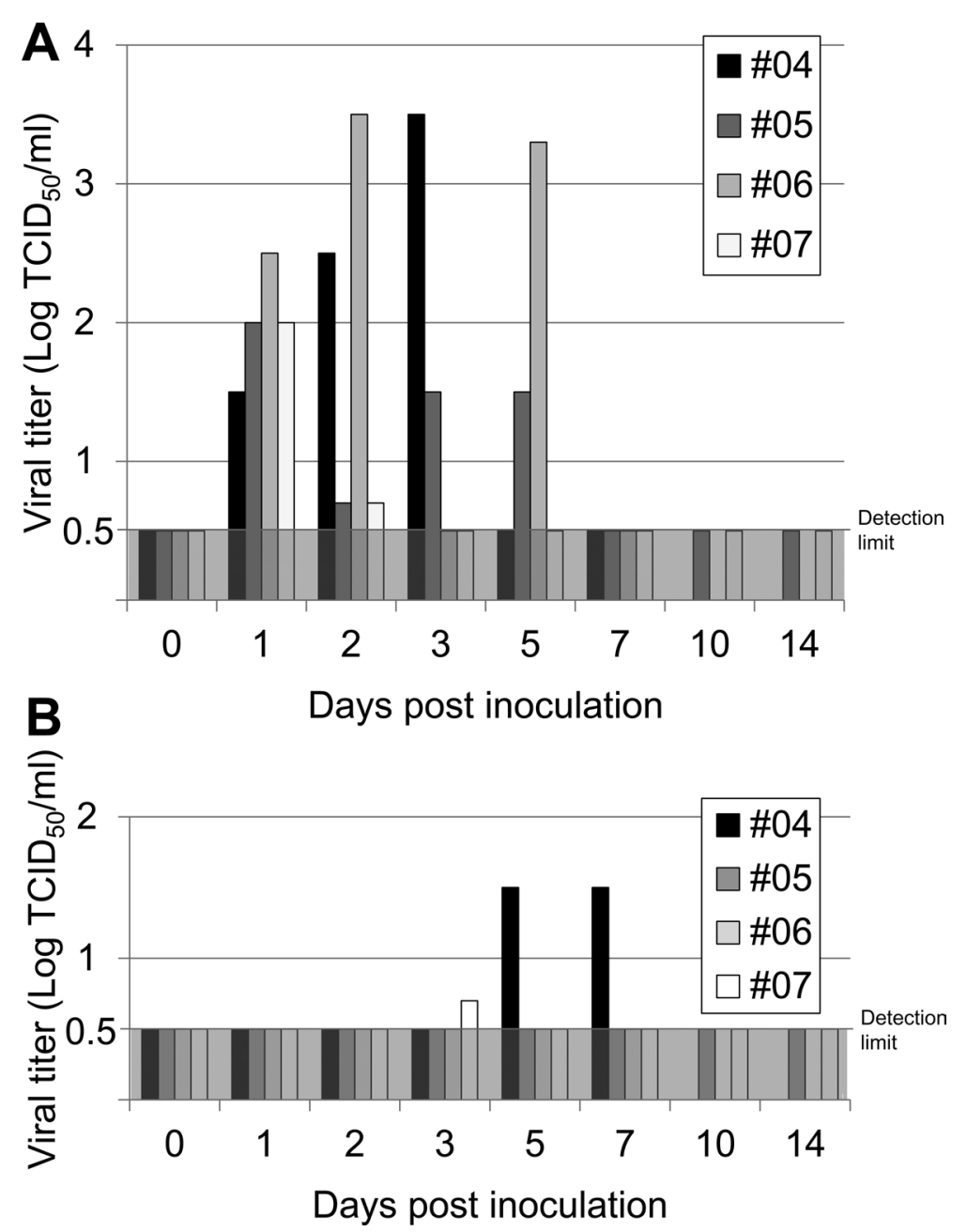

Fig. 2. Virus titers of laryngopharyngeal (A) and cloacal (B) swabs collected from the mandarin ducks in Group 2. Viral titers were calculated by the method of Reed and Muench [11] and are expressed as $\mathrm{TCID}_{50}$ per milliliter of the samples.

chea, corresponding to the virus shedding in laryngopharyngeal swab samples of the birds in Group 2 at the early stage of infection (Figs. 2 and 3). The viruses were also recovered from the kidney and the blood (Fig. 3), indicating that systemic infection could occur in mandarin ducks. In this study, the birds did not show any neurological symptoms, and no virus was isolated from the brains. It was previously reported that histological lesions and viral antigen were observed in the brain of a mandarin duck that died of H5N1 HPAIV infection [8]. This discrepancy may be because the strains used in this study and the previous study had different tissue tropisms. In the ducks that died at the late phase of infection, viruses were recovered from the lung, kidney and colon (Fig. 3). Therefore, dead mandarin ducks probably act as a source of infection to birds of prey and carnivores (e.g., Falconiformes [1, 12, 19] and raccoons [2]), which occasionally eat carrion.
Together, our results suggest that the recent $\mathrm{H} 5 \mathrm{~N} 1$ HPAIVs circulating in East Asia are lethal to mandarin ducks. To prevent future HPAI outbreaks, attempts should be made to keep wild birds out of poultry farms. Furthermore, the number and species of wild birds around farms need to be continuously monitored to reduce the risk of viral transmission to poultry populations.

ACKNOWLEDGMENTS. We thank Inokashira Park Zoo for providing the mandarin ducks, and Mr. J. Nakamori for giving technical advice about aviculture. This study was supported by the program of Strategic Fund for Promotion of Science and Technology of the Ministry of Education, Culture, Sports, Science and Technology, Japan. 


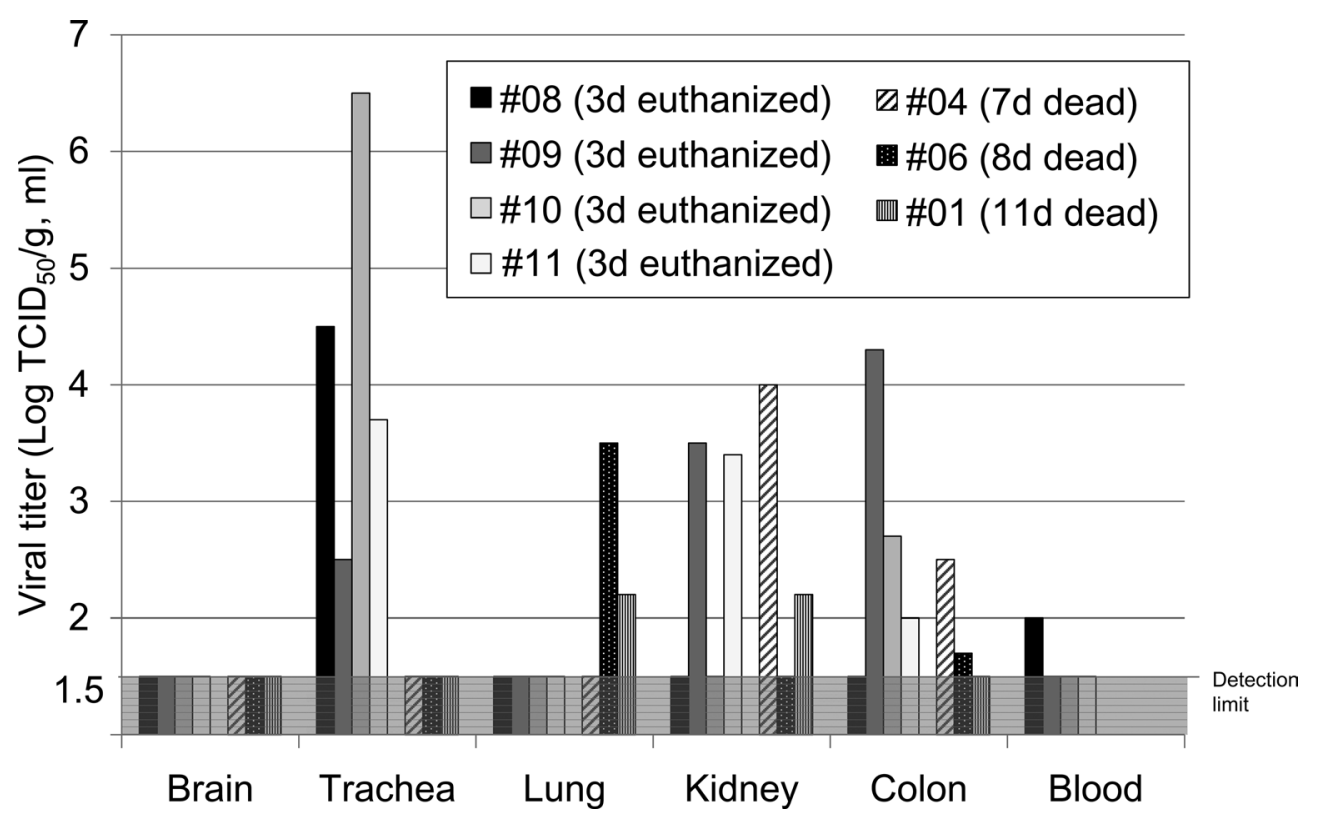

Fig. 3. Virus titers of the tissues and the blood collected from the euthanized mandarin ducks in Group 3 and the other birds died of virus infection. Viral titers were calculated by the method of Reed and Muench [11] and are expressed as $\mathrm{TCID}_{50}$ per gram and milliliter of the tissue and the blood, respectively.

\section{REFERENCES}

1. Couacy-Hymann, E., Danho, T., Keita, D., Bodjo, S. C., Kouakou, C., Koffi, Y. M., Beudje, F., Tripodi, A., De Benedictis, P. and Cattoli, G. 2009. The first specific detection of a highly pathogenic avian influenza virus (H5N1) in Ivory Coast. Zoonoses Public Health 56: 10-15. [Medline] [CrossRef]

2. Horimoto, T., Maeda, K., Murakami, S., Kiso, M., IwatsukiHorimoto, K., Sashika, M., Ito, T., Suzuki, K., Yokoyama, M. and Kawaoka, Y. 2011. Highly pathogenic avian influenza virus infection in feral raccoons, Japan. Emerg. Infect. Dis. 17: 714-717. [Medline] [CrossRef]

3. Jutglar, F. 1992. Handbook of the Birds of the World. Vol. 1. pp.596-599. In: Plate 44 ANATIDAE V (del Hoyo, J., Elliott, A. and Sargatal, J. eds.), Lynx Edicions, Barcelona.

4. Kajihara, M., Matsuno, K., Simulundu, E., Muramatsu, M., Noyori, O., Manzoor, R., Nakayama, E., Igarashi, M., Tomabechi, D., Yoshida, R., Okamatsu, M., Sakoda, Y., Ito, K., Kida, H. and Takada, A. 2011. An H5N1 highly pathogenic avian influenza virus that invaded Japan through waterfowl migration. Jpn. J. Vet. Res. 59: 89-100. [Medline]

5. Kida, H., Brown, L. E. and Webster, R. G. 1982. Biological activity of monoclonal antibodies to operationally defined antigenic regions on the hemagglutinin molecule of A/Seal/Massachusetts/1/80 (H7N7) influenza virus. Virology 122: 38-47. [Medline] [CrossRef]

6. Kim, H. R., Kim, B. S., Bae, Y. C., Moon, O. K., Oem, J. K., Kang, H. M., Choi, J. G., Lee, O. S. and Lee, Y. J. 2011. H5N1 subtype highly pathogenic avian influenza virus isolated from healthy mallard captured in South Korea. Vet. Microbiol. 151: 386-389. [Medline] [CrossRef]

7. Kim, H. R., Lee, Y. J., Park, C. K., Oem, J. K., Lee, O. S., Kang, H. M., Choi, J. G. and Bae, Y. C. 2012. Highly pathogenic avian influenza $(\mathrm{H} 5 \mathrm{~N} 1)$ outbreaks in wild birds and poultry, South Korea. Emerg. Infect. Dis. 18: 480-483. [Medline] [CrossRef]
8. Kwon, Y. K., Thomas, C. and Swayne, D. E. 2010. Variability in pathobiology of South Korean H5N1 high-pathogenicity avian influenza virus infection for 5 species of migratory waterfowl. Vet. Pathol. 47: 495-506. [Medline] [CrossRef]

9. Mase, M., Tsukamoto, K., Imada, T., Imai, K., Tanimura, N., Nakamura, K., Yamamoto, Y., Hitomi, T., Kira, T., Nakai, T., Kiso, M., Horimoto, T., Kawaoka, Y. and Yamaguchi, S. 2005. Characterization of $\mathrm{H} 5 \mathrm{~N} 1$ influenza A viruses isolated during the 2003-2004 influenza outbreaks in Japan. Virology 332: 167-176. [Medline] [CrossRef]

10. Ministry of Agriculture Forestry and Fisheries Japan 2011. Epidemiological investigation report of highly pathogenic avian influenza in 2010-2011. http://www.maff.go.jp/j/syouan/douei/ tori/pdf/ai_report.pdf (in Japanese).

11. Reed, M. and Muench, H. 1938. A simple method for estimating fifty percent endpoints. Am. J. Hyg. 37: 493-497.

12. Sakoda, Y., Ito, H., Uchida, Y., Okamatsu, M., Yamamoto, N., Soda, K., Nomura, N., Kuribayashi, S., Shichinohe, S., Sunden, Y., Umemura, T., Usui, T., Ozaki, H., Yamaguchi, T., Murase, T., Ito, T., Saito, T., Takada, A. and Kida, H. 2012. Reintroduction of $\mathrm{H} 5 \mathrm{~N} 1$ highly pathogenic avian influenza virus by migratory water birds, causing poultry outbreaks in the 2010-2011 winter season in Japan. J. Gen. Virol. 93: 541-550. [Medline] [CrossRef]

13. Shivakoti, S., Ito, H., Otsuki, K. and Ito, T. 2010. Characterization of $\mathrm{H} 5 \mathrm{~N} 1$ highly pathogenic avian influenza virus isolated from a mountain hawk eagle in Japan. J. Vet. Med. Sci. 72: 459-463. [Medline] [CrossRef]

14. Soda, K., Ozaki, H., Sakoda, Y., Isoda, N., Haraguchi, Y., Sakabe, S., Kuboki, N., Kishida, N., Takada, A. and Kida, H. 2008. Antigenic and genetic analysis of H5 influenza viruses isolated from water birds for the purpose of vaccine use. Arch. Virol. 153: 2041-2048. [Medline] [CrossRef]

15. Sugimura, T., Ogawa, T., Tanaka, Y. and Kumagai, T. 1981. Antigenic type of fowl plague virus isolated in Japan in 1925. Natl. 
Inst. Anim. Health Q (Tokyo) 21: 104-105. [Medline]

16. Tanimura, N., Tsukamoto, K., Okamatsu, M., Mase, M., Imada, T., Nakamura, K., Kubo, M., Yamaguchi, S., Irishio, W., Hayashi, M., Nakai, T., Yamauchi, A., Nishimura, M. and Imai, K. 2006. Pathology of fatal highly pathogenic H5N1 avian influenza virus infection in large-billed crows (Corvus macrorhynchos) during the 2004 outbreak in Japan. Vet. Pathol. 43: 500-509. [Medline] [CrossRef]

17. Uchida, Y., Mase, M., Yoneda, K., Kimura, A., Obara, T., Kumagai, S., Saito, T., Yamamoto, Y., Nakamura, K., Tsukamoto, K. and Yamaguchi, S. 2008. Highly pathogenic avian influenza virus (H5N1) isolated from whooper swans, Japan. Emerg. Infect. Dis. 14: 1427-1429. [Medline] [CrossRef]

18. Uchida, Y., Suzuki, Y., Shirakura, M., Kawaguchi, A., Nobusawa, E., Tanikawa, T., Hikono, H., Takemae, N., Mase, M., Kanehira, K., Hayashi, T., Tagawa, Y., Tashiro, M. and Saito, T. 2012. Genetics and infectivity of H5N1 highly pathogenic avian influenza viruses isolated from chickens and wild birds in Japan during 2010-11. Virus Res. 170: 109-117. [Medline] [CrossRef]
19. Van Borm, S., Thomas, I., Hanquet, G., Lambrecht, B., Boschmans, M., Dupont, G., Decaestecker, M., Snacken, R. and Van Den Berg, T. 2005. Highly pathogenic H5N1 influenza virus in smuggled Thai eagles, Belgium. Emerg. Infect. Dis. 11: 702-705. [Medline] [CrossRef]

20. World Organisation for Animal Health 2012. Web portal on Avian Influenza, Animal health in the World, http://www.oie.int/ animal-health-in-the-world/web-portal-on-avian-influenza/.

21. Xu, X., Subbarao, Cox, N.J. and Guo, Y. 1999. Genetic characterization of the pathogenic influenza A/Goose/Guangdong/1/96 (H5N1) virus: similarity of its hemagglutinin gene to those of H5N1 viruses from the 1997 outbreaks in Hong Kong. Virology 261: 15-19. [Medline] [CrossRef]

22. Yamamoto, N., Sakoda, Y., Motoshima, M., Yoshino, F., Soda, K., Okamatsu, M. and Kida, H. 2011. Characterization of a nonpathogenic $\mathrm{H} 5 \mathrm{~N} 1$ influenza virus isolated from a migratory duck flying from Siberia in Hokkaido, Japan, in October 2009. Virol. J. 8: 65. [Medline] [CrossRef] 